\title{
From (Un)Willingness to InvolveMENt: Development of a Successful Study Brand for Recruitment of Diverse MSM to a Longitudinal HIV Research
}

\author{
Paula M. Frew, ${ }^{1,2,3,4}$ Victoria A. Williams, ${ }^{1}$ Eve T. Shapiro, ${ }^{1}$ Travis Sanchez, ${ }^{2,5}$ \\ Eli S. Rosenberg, ${ }^{2,5}$ Vincent L. Fenimore, ${ }^{6}$ and Patrick S. Sullivan ${ }^{2,5}$ \\ ${ }^{1}$ Department of Behavioral Sciences and Health Education, Rollins School of Public Health, Emory University, \\ Atlanta, GA 30322, USA \\ ${ }^{2}$ Emory Center for AIDS Research, Atlanta, GA 30322, USA \\ ${ }^{3}$ Division of Infectious Diseases, Department of Medicine, Emory University School of Medicine, Atlanta, GA 30322, USA \\ ${ }^{4}$ The Hope Clinic of the Emory Vaccine Center, Decatur, GA 30030, USA \\ ${ }^{5}$ Department of Epidemiology, Rollins School of Public Health, Emory University, Atlanta, GA 30322, USA \\ ${ }^{6}$ School of Education, Georgia State University, Atlanta, GA 30303, USA
}

Correspondence should be addressed to Paula M. Frew; pfrew@emory.edu

Received 31 July 2013; Revised 11 November 2013; Accepted 21 November 2013

Academic Editor: Neil T. Hanlon

Copyright ( $\odot 2013$ Paula M. Frew et al. This is an open access article distributed under the Creative Commons Attribution License, which permits unrestricted use, distribution, and reproduction in any medium, provided the original work is properly cited.

Background. HIV continues to be a major concern among MSM, yet Black MSM have not been enrolled in HIV research studies in proportionate numbers to White MSM. We developed an HIV prevention research brand strategy for MSM. Methods. Questionnaires and focus groups were conducted with 54 participants. Descriptive statistics and chi-square analyses were performed and qualitative data were transcribed and content analyzed to identify common themes. Results. Formative research results indicated that younger Black MSM (18-29 years) were less likely to think about joining prevention studies compared to older ( $\geq 30$ years) Black MSM $\left(x^{2}=5.92, P=0.015\right)$. Qualitative and quantitative results indicate four prominent themes related to brand development: (1) communication sources (message deliverer), (2) message (impact of public health messaging on perceptions of HIV research), (3) intended audience (underlying issues that influence personal relevance of HIV research), and (4) communication channels (reaching intended audiences). Conclusion. The findings highlight the importance of behavioral communication translational research to effectively engage hard-to-reach populations. Despite reservations, MSM in our formative study expressed a need for active involvement and greater education to facilitate their engagement in HIV prevention research. Thus, the brand concept of "InvolveMENt" emerged.

\section{Introduction}

An estimated 1.1 million individuals, diagnosed and undiagnosed, have HIV in the United States [1]. Individuals of ages 20-24 (36.4 per 100,000) and 25-29 (35.4 per $100,000)$ have higher diagnosis rates than any other age category [1]. In 2010, there were 48,078 new diagnoses of HIV infection among adults and adolescents [1]. Of those cases, the majority were men $(37,910 ; 79 \%)$ [1]. Seventy-seven percent of those men reported male-to-male sexual contact as the risk-factor that attributed to their diagnosis [1]. In 2011, Black/African Americans carried the largest burden of disease with a new diagnosis rate of 60.4 per 100,000, followed by Hispanic/Latinos $(19.5$ per 100,000$)$ and Whites $(7.0$ per 100,000) [2]. Moreover, Black men who have sex with men (MSM) are disproportionately affected by HIV than any other group [2].

The southern part of United States (comprised of Alabama, Arkansas, Delaware, District of Columbia, Florida, Georgia, Kentucky, Louisiana, Maryland, Mississippi, North Carolina, Oklahoma, South Carolina, Tennessee, Texas, Virginia, and West Virginia) has the highest HIV prevalence rate per 100,000 than any other region [2]. In the South, 20.9 cases per 100,000 persons were newly diagnosed with HIV compared 
to only $18.1 \mathrm{HIV}$ cases per 100,000 in the Northeastern US (Connecticut, Maine, Massachusetts, New Hampshire, New Jersey, New York, Pennsylvania, Rhode Island, and Vermont), 12.0 HIV cases per 100,000 in the West (Alaska, Arizona, California, Colorado, Hawaii, Idaho, Montana, Nevada, New Mexico, Oregon, Utah, Washington, and Wyoming), and 9.3 cases per 100,000 in the Midwest (Illinois, Indiana, Iowa, Kansas, Michigan, Minnesota, Missouri, Nebraska, North Dakota, Ohio, South Dakota, and Wisconsin) [2]. Furthermore, the greatest proportion of Black/African Americans (55\%) resides in the South [3]. In 2011, African Americans constituted 64.1 per 100,000 cases of all new HIV diagnoses. This represents a disproportionate amount of cases based on the overall 20.9 per 100,000 case rate in the South [2]. In Georgia, Blacks make up $31.5 \%$ of the population [3]. Georgia $(2,522)$ was ranked number 5 after California $(5,973)$, Florida (5,403), Texas $(5,065)$, and New York $(4,960)$ for having the highest number of new HIV diagnoses in 2011 [1]. The disproportionately high apparent incidence of HIV in the South, among Blacks and among MSM, establishes Atlanta as a prime location for conducting formative HIVrelated research to understand how to include disproportionately impacted groups in research. These groups may be populations of which novel HIV prevention and treatment advances may be a benefit to stem transmission rates among these affected groups.

1.1. HIV Research Recruitment. There is a limited amount of reported data on HIV research brand formulation and development of HIV recruitment, outreach, and communication approaches. [4] Cultivating a successful study brand identity - the set of characteristics that distinguishes a brand, such as a logo or slogan-and brand personality - the way a brand is marketed so that the target group can relate to it-requires an understanding of the values of the target group which is often elicited via qualitative methods such as focus groups and observation $[5,6]$. These values are articulated through messages that garner attention and foster interest in participation [7-9]. With an active pipeline of longitudinal HIV studies planned and underway with MSM, there is a need to understand how to effectively develop recruitment campaigns to achieve success with future HIV research recruitment endeavors. Moreover, understanding how to build a study brand that attracts the attention, interest, and participation of diverse MSM may provide guidance to other HIV prevention campaigns, particularly those promoting HIV testing, linkage-to-care, and other behavioral and biomedical approaches for MSM $[10,11]$. As we planned to utilize venue-based sampling for the longitudinal study, we recognized the need to develop a brand that would enable our team to immediately connect with potential participants as the recruitment strategy did not enable us to advertise the study to the target population [12]. As the venue-based approach requires not only randomization of venue sites to conduct recruitment but also times for recruitment, this strategy of encountering people at the "right place, right time" necessitates an identification with the study, the team, and the values underlying the research endeavor to promote their willingness to join the study [12-14].
1.2. Conceptual Dimensions. Persuasive communication theory suggests that attitudes and beliefs are influenced by the interplay of variables as the audience (recipient) evaluates a message and source within a specific context (e.g., deciding whether to participate in HIV research) [15-17]. The elaboration likelihood model's focus on the relationship of motivational attributes (e.g., components of a brand that trigger either an emotional response or help to form a relationship to the product) with the receiver's ability to process information provides a useful conceptual framework for content analysis of recruitment campaigns $[18,19]$. Application of the model would suggest that those who consider participating in an HIV research would face a risk-taking decision and therefore may engage in careful thinking about the study information (e.g., "do I think this will benefit me or my community and what is the risk to me if I participate at this point?"). This high degree of consideration before enrollment (i.e., "high involvement" processing) would theoretically permit counter persuasion to occur in response to any negative stimuli (e.g., friends and family's negative reactions toward participation). A persuasive brand would however overcome any experienced negative reactions in the decisional process to motivate individuals toward the behavioral goal (e.g., study enrollment).

Our study sought to understand the persuasive influences that should be incorporated into a brand strategy (including the recruitment campaign) as there is a dearth of information guiding HIV/AIDS clinical/behavioral research studies [2022]. The objective of our study was to specifically develop an effective brand to successfully enroll MSM into our longitudinal HIV study that relied on venue-based sampling to efficiently recruit the population in a time-limited period. The objective of our longitudinal ("InvolveMENt") study was to explaining disparities in HIV prevalence and incidence in Black and White MSM by examining the effects of dyadic, network, and community-level factors on the HIV risk of Black MSM compared to their White counterparts. Therefore, in an environment where HIV studies recruiting MSM are many, we needed a brand that could compete against advertised studies in places they frequented such as clubs, bars, gyms and spas, and other locations. Our approach conformed to the development of a public health communication campaign that is considered to be focused in its aims, time-limited, and conducted within an organized, coherent manner [23]. Because the decision to participate in an HIV research study may be promoted by factors aligned with the conceptual pathways such as message content, issue relevance, and source and situational cues (i.e., heuristics), our study highlights how we evaluated these issues to create a successful strategy for the successful enrollment of 803 men (454 Black, 349 White) in the longitudinal "InvolveMENt" study from July 2010 to December 2012.

\section{Methods}

2.1. Data Collection. The formative study was conducted from late 2009 through early spring 2010, prior to launching venue-based recruitment in July 2010 for the longitudinal "InvolveMENt" study. A mixed methods approach included 
the conduct of focus groups with accompanying questionnaires gathered at each session. In addition, to direct recruitment via web advertisements, partner organizations facilitated recruitment of men from a variety of community settings to ensure broad sociodemographic representation of Black and White MSM. Six focus groups were conducted with 5 to 13 MSM and transgender (male to female) participants in each group. The focus groups took place at diverse communications locations in Atlanta, Georgia.

2.2. Instruments. Detailed instruments were developed to gather information on a number of issues that would inform the development of our brand strategy. Topics covered in the focus groups included, but were not limited to, social networks, online behavior, nature and patterns of communication, communication issues, current HIV prevention messaging, HIV/AIDS research, clinical trials, and HIV vaccine studies. Questionnaires gathered information on sociodemographics, HIV communication recall, and the extent of media consumption for health information topics. We specifically evaluated components of the ELM model that are theorized as key operants in persuasion, including personal relevance, message framing, message components, and source characteristics.

We examined ELM operants to inform our brand development approach via mixed methodologies. Qualitative results support the quantitative findings inclusive of the following themes: (1) communication source: credible sources who will ameliorate perceptions of HIV research (e.g. dangerous, scary, etc.), (2) message: participation barriers and motivators (e.g. compensation), (3) intended audience: underlying issues that influence participation of target audiences (e.g. personal histories, gay rights issues), and (4) communication channels: the need for targeted communication in appropriate venues with intended audiences during research phases (e.g., social media).

2.3. Communication Sources. According to ELM, persuasive appeals have the potential to alter behavior with messages that invoke particular types of meanings and relevant symbolic representations [24]. Although "central processing" may be affected by motivation and ability to attend to the message as issue-relevant thinking is generated [19], previous studies have indicated that heuristic cues may favorably shape attitudes towards participation in clinical research through "peripheral processing" routes [20] and have the potential to generate strong reactions to HIV/AIDS prevention messages [22]. We therefore elicited information on the cues to the formation of attitude toward potential brand messages (e.g., site and investigator details).

2.4. Message. For this ELM operant, we explored the amount of information contained in the items about HIV/AIDS risk because of the potential to capture attention [25-27] and engage the target audience through the personal relevance of the health issue. Ads with risk messages have been shown to be effective [25], but they may also invoke protective cognitive appraisal [27]. In this context, greater involvement with the issues of HIV/AIDS research theoretically lends to "central route" message processing in the promotion of attitude change $[15,19]$. In the ELM model, "central route" processing engages more thoughtful processing rather than the emotional response elicited by the "peripheral route." [28] Communication approaches that address these concerns may enable central route processing to occur via evaluation of important issues that may hold relevance in the formation of attitudes. We therefore assessed the nature of benefits extended to prospective study participants that may encourage enrollment as well as barriers to participation.

2.5. Intended Audience. Language and visuals attesting to those involved in the research may serve as motivational cues for MSM as well as other audiences. Recruiters, community members, and study spokespersons alter the persuasive nature of the brand. Whether a brand is considered trustworthy often depends on language and depictions presented. Thus, we evaluated how to achieve source credibility. We accomplished this by asking men who need to develop the messages, what person(s) need to promote the (study) brand, and what visuals should be present in the campaign to garner their attention.

2.6. Communication Channels. With very limited resources allocated to recruitment efforts given our venue-based recruitment strategy, an assessment of potential opportunities-to-see (OTS) at various sites was of interest to this study. In particular, we were interested in where men were obtaining information to determine if recruitment sites could be expanded to online venues. Thus, we created a media consumption instrument to evaluate the communication channels relevant to men's needs and how to leverage social media for optimal recruitment and engagement with the emergent brand identity.

2.7. Participants. Participants were recruited from a variety of venues including bars, clubs, restaurants, gyms, and online sites via advertisements. Participants were eligible for inclusion if they reported (1) being male by birth, (2) being nonHispanic White or Black/African American, (3) oral or anal sex in the past six months with a male partner, and (4) live in the metropolitan Atlanta area. Men and transgender women were compensated $\$ 25$ for their time to attend the sessions.

2.8. Data Analysis. Descriptive statistics, chi-square analyses, and factor analyses were performed on quantitative data from focus group participants $(N=54)$ using SPSS. Qualitative data were transcribed by a member of the team. The data were independently coded by two coders and content analyzed to identify common themes related to participating in HIV prevention research studies. A random sample of transcripts (20\%) was cross-coded for reliability. The presence or absence of codes from a subsample of interviews was compared using qualitative software. The overall reliability of the sample was 92\% (which is above the suggested 90\% agreement level) [29]. The coding was completed from February 2013 to March 2013.

2.9. Codebook Development. A detailed codebook was developed from focus group transcripts. The original codebook was modified as other codes emerged from coding other 
transcripts. The final codebook was created after reviewing all of the transcripts and discussing emergent themes with members of the research team. Coding schemes were developed to capture relevant details from the transcripts such as attitudes, beliefs, and opinions about participating in HIV research. The final codebook contained variables organized within the following rubric of four major themes: (1) intended audience: underlying issues that influence participation of target audiences (e.g., personal histories, gay rights issues), (2) message: Participation barriers and motivators (e.g., compensation), (3) source: credible sources who will ameliorate perceptions of HIV research (e.g., dangerous, scary, etc.), and (4) communication channels: the need for targeted communication in appropriate venues with intended audiences during research phases (e.g., social media).

\section{Results}

3.1. Participant Characteristics. A total of 54 individuals including 53 MSM and 1 male-to-female transgender woman participated in the focus groups and completed the questionnaire (Table 1). The mean age of the study participants was 31 years (range: $18-39, \mathrm{SD}=8.7$ years). All of the participants reported having at least a high school education and the majority had at least a bachelor's degree $(67 \%, n=36)$. Fifty-one self-identified as non-Hispanic (94\%); three were Hispanics (6\%). The majority reported their race as Black $(70 \%, n=38)$, White $(24 \%, n=13)$, or identifying as White and/or Black with a "multiracial/multicultural" heritage $(6 \%$, $n=3)$. All participants identified as either homosexual (93\%, $n=50)$ or bisexual $(7 \%, n=4)$. Eighty-five percent $(n=$ 46) were single, $13 \%(n=7)$ were married/in a domestic partnership, and 1 individual reported that he was divorced/ separated $(2 \%)$. Half ( $n=27,51 \%)$ were employed full-time and most participants earned less than $\$ 40,000$ in their work $(n=45,83 \%)$.

3.2. Communication Sources. Issues related to those conducting the study and messages conveyed by persons or research entities (sources) revealed that a welcoming feeling at the study site was of importance to Black MSM when determining whether they should consider participating in HIV research $\left(x^{2}=3.93, P=0.047\right)$. Only Black participants $(n=6,11 \%)$ selected the response "not enough of my people running the study" when asked "what concerns do you have about joining an HIV prevention study?” Conversely, Blacks $(n=33,87 \%)$ were "very likely" to participate in a study if the study staff were viewed as welcoming compared to Whites $(n=8,61.5 \%)$. Additionally, the importance of having "a track record of success with previous clinical studies at the study site" was of greater importance to Black MSM compared to Whites $\left(x^{2}=12.92, P=0.002\right)$. More Black participants were either "very likely" ( $n=26,68 \%)$ or "somewhat likely" $(n=11,29 \%)$ to participate than White participants $(n=8$, $61.5 \%)$ if this criteria were met.

In the focus groups, men identified factors that influence their decision to participate in HIV prevention research. Many felt that there are many misconceptions about research and therefore selecting the right source for message delivery
TABLE 1: Involvement formative research participant sociodemographics $(N=54)$.

\begin{tabular}{|c|c|c|}
\hline & Frequency & Percent \\
\hline \multicolumn{3}{|l|}{ Gender } \\
\hline Male & 53 & $98 \%$ \\
\hline Transgender (male to female ) & 1 & $2 \%$ \\
\hline Mean age (range) & $31(\mathrm{SD}=8.7)$ & $18-39$ \\
\hline \multicolumn{3}{|l|}{ Highest level of education } \\
\hline High school & 12 & $22 \%$ \\
\hline Technical/vocational or associates & 6 & $11 \%$ \\
\hline Bachelor degree & 26 & $48 \%$ \\
\hline Master's degree & 9 & $17 \%$ \\
\hline Doctorate & 1 & $2 \%$ \\
\hline \multicolumn{3}{|l|}{ Ethnicity } \\
\hline Hispanic & 3 & $6 \%$ \\
\hline Non-Hispanic & 51 & $94 \%$ \\
\hline \multicolumn{3}{|l|}{ Race (missing $n=1$ ) } \\
\hline African American/Black & 38 & $70 \%$ \\
\hline Caucasian/white & 13 & $24 \%$ \\
\hline Multiracial/multicultural & 1 & $2 \%$ \\
\hline Other & 1 & $2 \%$ \\
\hline \multicolumn{3}{|l|}{ Sexual orientation } \\
\hline Gay/homosexual/queer/questioning & 50 & $93 \%$ \\
\hline Bisexual & 4 & $7 \%$ \\
\hline \multicolumn{3}{|l|}{ Relationship status } \\
\hline Single & 46 & $85 \%$ \\
\hline Married/domestic partner & 7 & $13 \%$ \\
\hline Divorced/separated & 1 & $2 \%$ \\
\hline \multicolumn{3}{|l|}{ Work status } \\
\hline Employed full-time & 27 & $50 \%$ \\
\hline Employed part-time & 14 & $26 \%$ \\
\hline Unemployed & 10 & $19 \%$ \\
\hline Other & 3 & $6 \%$ \\
\hline \multicolumn{3}{|l|}{ Annual household income } \\
\hline Less than $\$ 20,000$ & 20 & $37 \%$ \\
\hline$\$ 20,001-\$ 40,000$ & 25 & $46 \%$ \\
\hline$\$ 40,001-\$ 60,000$ & 5 & $9 \%$ \\
\hline$\$ 60,001-\$ 80,000$ & 1 & $2 \%$ \\
\hline More than $\$ 100,000$ & 3 & $6 \%$ \\
\hline \multicolumn{3}{|l|}{ Primary healthcare facility } \\
\hline Healthcare provider office & 33 & $61 \%$ \\
\hline Community clinic or health center & 14 & $26 \%$ \\
\hline Hospital & 5 & $9 \%$ \\
\hline Other location & 2 & $4 \%$ \\
\hline
\end{tabular}

is critical. Additionally, information must be conveyed in a manner that considers not only the health literacy of the population but also the cultural relevance of studies. One man revealed

...given the opportunity and presented to them it may be, but a lot of people just do not know about it or know what's going on or feel indifferent because they do not understand the importance 
because it's never been presented to them in a way to make them understand that this is what's going on, this is why we're doing this and this is important to us.

We also explored the sources of information in the community that convey information about research as many Black MSM expressed reluctance to participate based on their knowledge of historical events, such as the Tuskegee experiment.

Um we think about the Tuskegee Institute and what people are taught from grandparents and great grandparents about what that was and what the facts that they understood at the time were versus what the reality of the facts are, um, and where these have gone since then.

We learned that research stories have been passed from generation to generation among Black families. Therefore, acknowledging the oral tradition is important while correcting facts about what actually happened in previous studies. During these discussions, the White MSM did not respond on the issue of historical events and oral traditions. This evidenced the importance of developing a nuanced brand that would achieve resonance among these groups to successfully recruit a diverse group of participants. Thus, the source of information must be highly knowledgeable and trusted by the community:

\section{I will say that in general, my experience dealing with the African American community is that there's a certain fear of clinical trials um and it's less based on historical fact more based on histor- ical inaccuracies that have been passed down.}

3.3. Messaging. Participants expressed a high level of "prevention message" fatigue on assessed items including safer sex messages and related materials $(r=0.895, P<0.001)$ and general HIV campaigns $(r=0.699, P<0.001)$ and condom use in HIV prevention campaigns $(r=0.444, P=0.005)$. Factor analyses resulted in the development of the 5-item "message fatigue" scale (Eigenvalue $=3.166,63 \%$ variance) with high internal consistency (Cronbach alpha $=0.842)$. We specifically examined any potential differences in attitudes about prevention messaging by age and race; however, we yielded nonsignificant results on these analyses. When asked about messaging specifically, "[are] messages about safe sex. . beginning to sound the same?" most men $(n=34,63 \%)$ agreed or strongly agreed with the statement. The focus group conversation also highlighted the perceived ineffectiveness of current HIV prevention messages for this population. Men expressed a need for different kinds of messages that would be particularly relevant to younger MSM. One participant summarized the group sentiment:

\section{If you just look at the statistics um increasing infection rates, clearly the tools that are out there are not effectively used to prevent $H I V$.}

We explored the extent to which this perception is also held about prevention methods such as condom use and safer sex messages. The men shared agreement ( $n=24,45 \%)$ that "people I know are burned out on safe sex campaigns." Additionally, we explored responses to the question, "after seeing them for years, safe sex brochures and pamphlets seem repetitive" to discern how to communicate information about the study to potential participants. The majority expressed agreement with this statement $(n=36,64 \%)$ thus signaling that new forms of nonprint communication may be necessary for this audience.

In focus groups, men repeatedly stated that improved tactics should be utilized to convey messages to MSM. They also discussed the value of personal narrative as a messaging approach as opposed to messages conveyed by health professionals. Thus, the men indicated a need for communitycentered messages to promote research involvement. One stated

You know because people are...they're not... they're thinking levels are different and they're on different levels so, if a friend of mine personally went through what they went through and told me, I hope I'd believe them versus a doctor...

3.4. Intended Audience. The majority $(n=39,72 \%)$ of participants reported that they had not been in a clinical research study. Previous clinical research study participation differed significantly between older ( $\geq 30$ years) and younger (18-29 years) MSM $\left(x^{2}=4.16, P=0.041\right)$. Older MSM are more likely to have participated in a study in the past. Despite reported challenges of recruiting Black participants, Blacks were more likely to have been in a clinical research study than White MSM $\left(x^{2}=6.39, P=0.011\right)[30]$. However, within the Black MSM group, younger Black MSM (18-29 years) were less likely to think about joining prevention studies compared to older ( $\geq 30$ years) Black MSM $\left(x^{2}=5.92, P=0.015\right)$. Lack of time ( $n=15,28 \%)$, inconvenient location $(n=15$, $28 \%)$, and low compensation $(n=15,28 \%)$ were the top three intended audience barriers they perceived for recruitment into longitudinal HIV prevention studies.

Additionally, the participants were asked to describe events, experiences, and attitudes that would shape message perceptions conveyed by the research team to an intended MSM audience. Participants described the need to understand the process of coming "out" in the South, a process often occurring in relation to being intimately involved with others who were publically "out" with their own sexual orientation. Many underscored the fundamental need to belong and become involved in supporting causes, belonging to social networks, and joining friendly circles where sexual orientation is celebrated and not made an issue. Thus, the connection between their involvement in the MSM community and their identity became an important issue of discussion among men. One participant stated

\section{Um for me, no, it's very important for me to be out. And because I do value it so much as a part of my identity, so, someone said that they do not put it out there, like, I put it out there.}

Many of the participants indicated that their friends and other members of their social circles have varying sexual 
orientations (e.g., identifying as gay, straight, and bisexual). We found that younger men ( $\leq 30$ years) in the focus groups held different perceptions from older men on the subject of sexual identity. This did not seem to vary by race. One offered

I have friends who um, identify themselves very strictly. But a lot of people I know, um even men that I know exclusively have had sex with only men, they consider themselves more open or maybe bisexual because they're not closed to the idea of it. I have a lot of friends, being with a woman close to that, I have a lot of friends who, even if they've exclusively been with one gender, if they're open to the concept of meeting someone and being with another gender than what they would identify themselves with. I do have the ones that identify themselves more rigidly. But even, even straight male friends I have, sometimes say, well I have never been with a man, but I'm not closed to it if I was attracted. So a lot of the people I know really aren't very rigid in their self-identification. I think I would be a lot more than a lot of the people I know because I do consider myself strictly gay. But I mean a lot of people do have some grey area, even if they haven't experienced it.

Even though some talked about friends with sexual orientations that differ from their own, others would rather be around other gay men. One participant said

I prefer to hang out with gay men and people who
identify as gay men... I prefer to go to a gay bar,
I prefer to go to a gay restaurant, I prefer to read
gay publications, and even though I have straight
friends and I would certainly never, you know,
not accept someone's friendship, I do enjoy um
a camaraderie with people who identify, pretty
much exclusively, as gay men...I've preferred to
surround myself by gay men.

3.5. Communication Channels. We explored how we could reach diverse MSM in venues and through a variety of media sources. Black men expressed their need to be engaged in all aspects of study design, planning, and implementation. They indicated that they do not help to determine what, how, and who should communicate research information to them. Among the MSM, many conveyed a sense of frustration with communication researchers who come into the community with preformulated messages, communication materials, and dissemination approaches with the perceived intent to elicit target audience approval. One participant stated

I'm quite sure they had a focus group somewhere, something similar like this for their product...but people just saw it in the store that did not have any idea about it. . So like he was saying, if you put it out there and if they knew they could participate and have some say so about it. But most folks by the time something gets there you know they weren't the ones in the know or do they know the people who are in the know behind it.
Another participant identified mistrust of the individuals who conduct community-based research with MSM. Specifically, he underscored the need to be able to share their opinions in the research process:

I would be more interested in who's doing the data
analyzation, because sometimes I really do not
trust the people who analyze data or I do not
like their, I do not trust their perspectives. And
they sometimes come from a perspective that's not
similar to mine. And so I want to know, who's
going to be analyzing the data, and who's got kind
of similar ideas and perspectives like I do. Cause
I'm not going to waste my time, cause at the end
of the day, I do not want you skewing data to what
you want, so.

In conjunction with the focus group discussions on reaching MSM in venues and through other communication channels, we asked them to offer their "media consumption" preferences. Previously we found that younger men, including Black/African American men and those who identify as bisexual or heterosexual may not frequent physical venues frequented by MSM (e.g., bars or clubs) [14]. Thus, the need to identify other avenues, particularly those offered by online sites, to reach and interact with these populations was of interest to our team. The 53 persons who responded to our media questionnaire revealed an affinity for obtaining news from the Atlanta Journal Constitution $(n=32,60 \%), \mathrm{CNN}$ ( $n=16,30 \%)$, and The New York Times $(n=13,25 \%)$, as well as social media sites such as their top-ranking online venue, Facebook $(n=25,47 \%)$. Other local sources mentioned included Creative Loafing $(n=10,19 \%)$ and Southern Voice $(n=5,9 \%)$. Overall, participants indicated a preference for local, community-based news sources as well as global communication sources. Very few ranked LGBT-focused media outlets as preferred over mainstream sources thus signaling a desire to be reached through broader, inclusive communication platforms.

\section{Discussion}

We conducted a formative research study that utilized the ELM model to evaluate the significance of source, messaging, intended audience, and communication channels with 54 White/multiracial $(n=14,28 \%)$ and Black/African American $(n=38,72 \%)$ MSM. Consistent with the ELM framework, we found that all four elements were associated with the formation of attitudes toward participation in HIV prevention research [4].

Through our focus groups, our participants described components of the research process and study branding that paralleled existing findings from the literature. Black MSM especially were sensitive to feeling welcome and demographically represented by research staff as well as developing trust with staff throughout the study. They wanted to be included in multiple parts of the research process and that their input was incorporated in protocol development.

Previous qualitative and quantitative findings highlight the importance of selecting the right messenger (or "Communication Source") as HIV conspiracy theories and myths 
and misperceptions exacerbate the level of research distrust present in the community; this was especially notable among Black MSM [31]. Many Black participants stated that historical events, such as the Tuskegee experiment, influenced their decision to participate in studies $[31,32]$. Some Black MSM explicitly expressed their distrust for researchers; however, they stated that the inclusion of Black researchers would motivate their participation, particularly if the lead researcher was Black and served as an advocate for them [32]. Yet, men expressed in qualitative and quantitative components that the composition of the research team, and their demonstrated cultural competency, ultimately influenced their attitudes toward HIV study participation $[33,34]$. Thus, the selection of study team members who not only represented community but who also could articulate the importance and value of MSM in HIV research and demonstrate mutual investment was identified as ideal communication sources [34]. In short, men said they would believe messages from researchers who are viewed as credible, relatable, and trustworthy sources.

Our findings revealed that most MSM found that messages about HIV, condom promotion, and safer sex were no longer resonant with the population. Some expressed that they felt "burned out" on these topics and they described that the messages were beginning to sound the same. Other studies of MSM identified HIV prevention message fatigue similar to our findings [35]. Previous research with similar audiences has identified ELM cognitive processing as a contributor to message attentiveness [36]. Among younger persons, the findings indicate that the format and presentation of safe sex messages (cartoon and written) are mediated by their motivation to engage in and enjoy thinking about the material presented [36]. Thus, findings from the literature suggest HIV messages would be enhanced by understanding MSM's "information-processing proclivities" to maintain their relevance [36].

Our participants also discussed issues with promoting HIV research with MSM as the intended audience. Black men brought up concerns they had about being a Black MSM, including issues about coming out and being out in their community. The literature identified similar issues including feeling marginalized and disengaged from the community which leads to a profound sense of individual and group social and sexual isolation [37]. Such self-imposed exclusivity has been associated with increased community-level risk for HIV and STIs $[38,39]$. Black participants described that it is difficult to be a Black male and have the burden of a "second minority status" (MSM) yet retain a sense of resiliency and self-empowerment $[40,41]$. In addition to the challenges of exclusion in the Black community, Black MSM in our study articulated a divide with White MSM. Hence issues around inclusion, empowerment, and participation were brought to the forefront as themes that needed to be intertwined with the HIV research messages for this intended audience.

All of the men agreed that HIV research is important and needs to be conducted in order to cure or prevent HIV infections. Many stated that HIV has a direct impact in their personal and community life. We found that engaging MSM through appropriate communication channels was a final linkage in a successful brand strategy to connect people to research, conclusions further supported by previous studies $[5,6]$. In addition, men expressed the need to be a part of the preimplementation, implementation, and postimplementation phases of studies. In particular, their responses mirrored findings from other studies and highlighted the need to consider recruiting men from alternative venues such as internet sites in addition to typical MSM venues such as bars, clubs, gyms, and parks during the study planning period [42-44]. Yet, given their media preferences, our participants also cautioned against using exclusionary tactics to reach the audience online. They preferred Facebook and other local "mainstream" sites such as Creative Loafing online as venues for which study ads and recruitment could occur.

The lessons learned from this formative study underscore the need for extensive brand development research with diverse MSM audiences to distinguish the importance of HIV research and promote the relevance of their participation. Older men tended to be more open about their homosexuality compared to younger men which presented challenges for crafting a single brand that would be accepted across age groups. In addition to recognizing the fluidity around defining sexual identity, we recognized there were underlying reasons why men opted to frequent or stay away from physical venues we selected for recruitment. This was a particular challenge to consider in our brand development process for young Black MSM. We had to therefore remain sensitive in our brand wording about community connotations (e.g., being identified as gay by being at the place) as well as raciallyrelevant concerns such as being left behind in research decision-making. Thus, developing tailored communication messages for specific segments is the best practices approach. A strong brand will provide not only study recognition but also it provides the platform to convey many culturally congruent and resonant messages (i.e., unique taglines) and information to achieve recruitment goals.

In the end, we presented those in our sample with brand concepts of which the "InvolveMENt" concept was overwhelmingly selected over other options ("Your Move" and "Open-I") (Figure 1). Men indicated that InvolveMENt reflected themselves as central to achieving HIV prevention breakthroughs as strong MEN. It also built upon intersecting themes of the need for inclusion, individual empowerment, and community voice in HIV research.

\section{Conclusions}

The findings from this study significantly aided in the branding and recruitment strategy for subsequent HIV research projects. The preferred study title, "InvolveMENt" as well as the favored logo was utilized in a succeeding study. Recommendations from participants on staff demographics, developing trust, and encouraging participation throughout the study were utilized to develop a protocol that was intriguing and engaging to Black and White MSM. From July 2010 to December 2012, InvolveMENt enrolled 454 Black and 349 White MSM, achieving optimal enrollment of participants representative of the local MSM community and exceeding our initial recruitment goal of 660 persons. With an HIV incidence rate of $4.8 \%$ in Atlanta among Black MSM, 
Final selected brand concept used for InvolveMENt study

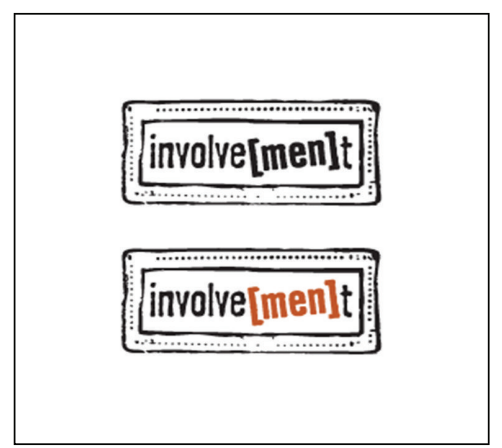

Alternative concept presented to participants

\section{MPEN・I

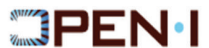

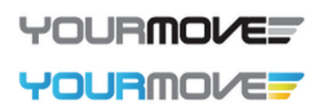

FIGURE 1: Selected study brand concepts presented to participants.

it is more critical than ever to recruit this population to HIV prevention interventions [45]. Yet, the difficulty of recruiting Black MSM in representative numbers for longitudinal HIV biomedical and prevention research studies continues to be an enduring problem [46-48]. However, using a culturallysensitive, inclusive brand development approach, the InvolveMENt study was able to exceed our recruitment goals, thus utilizing a theory-driven approach, such as ELM, to develop a project brand resulted in a persuasion strategy that became "InvolveMENt."

\section{Acknowledgments}

The authors acknowledge the support of National Institute of Mental Health R01MH085600, Minority Health and Health Disparities RC1MD004370, Eunice Kennedy Shriver National Institute for Child Health and Human Development R01HD067111, NIH P30AI050409 the Emory Center for AIDS Research, and the National Center for Advancing Translational Sciences of the National Institutes of Health under Award no. UL1TR000454. The authors wish to thank our participants and our partner organizations and their staff for supporting the programmatic activities associated with this work. Special thanks to Rick Kern, MixIt Marketing, for his leadership in brand development and its implementation for the InvolveMENt study.

\section{References}

[1] Centers for Disease Control and Prevention, "Diagnoses of HIV infection among adults and adolescents, by sex and transmission category, 2010-46 states and 5 U.S. dependent areas," HIV Surveillance Report 22, 2010.

[2] Centers for Disease Control and Prevention, "Diagnoses of HIV infection in the United States and dependent areas," HIV Surveillance Report 23, 2011.
[3] U. S. Census Bureau, The Black Population, 2010.

[4] P. M. Frew, W. Macias, and K. Chan, “'Step' with HIV vaccines? a content analysis of local recruitment campaigns for an international HIV vaccine Study," Journal of Health \& Mass Communication, vol. 1, no. 1, pp. 11-39, 2009.

[5] J. Hagerman, "Give them something to talk about. An integrated brand strategy keeps the focus on the patients, as the MedStar Health campaign races ahead," Marketing Health Services, vol. 32, no. 3, pp. 20-25, 2012.

[6] D. W. Miller, "A core strategy-developing a brand. A health care organization that builds strong brand loyalty will ensure its position for the future," Health Forum journal, vol. 44, no. 1, pp. 36-38, 2001.

[7] J. B. White and M. P. Miles, "The financial implications of advertising as an investment," Journal of Advertising Research, vol. 36, no. 4, pp. 43-52, 1996.

[8] L. Percy and R. Elliott, Strategic Advertising Management, vol. 335, Oxford University Press, Oxford, UK, 2nd edition, 2005.

[9] M. A. Allen, T. S. Liang, T. La Salvia, B. Tjugum, R. J. Gulakowski, and M. Murguía, "Assessing the attitudes, knowledge, and awareness of HIV vaccine research among adults in the United States," Journal of Acquired Immune Deficiency Syndromes, vol. 40, no. 5, pp. 617-624, 2005.

[10] D. M. Davison, G. D. Reeder, and K. Teverbaugh, "African-American volunteers carrying an HIV prevention message: selective communication," AIDS Education and Prevention, vol. 11, no. 5, pp. 436-449, 1999.

[11] J. Merrill, R. Kukafka, S. Bakken, R. Ferat, E. Agopian, and P. Messeri, "Tailored health communication: crafting the patient message for HIV TIPS," Proceedings of the AMIA Annual Symposium proceedings, p. 932, 2003.

[12] F. B. Muhib, L. S. Lin, A. Stueve et al., "A venue-based method for sampling hard-to-reach populations," Public Health Reports, vol. 116, supplement 1, pp. 216-222, 2001.

[13] S. M. Jenness, A. Neaigus, C. S. Murrill, C. Gelpi-Acosta, T. Wendel, and H. Hagan, "Recruitment-adjusted estimates of HIV prevalence and risk among men who have sex with men: effects of weighting venue-based sampling data," Public Health Reports, vol. 126, no. 5, pp. 635-642, 2011.

[14] T. Sanchez, A. Smith, D. Denson, E. Dinenno, and A. Lansky, "Internet-based methods may reach higher-risk men who have sex with men not reached through venue-based sampling," The Open AIDS Journal, vol. 6, pp. 83-89, 2012.

[15] R. E. Petty and J. T. Cacioppo, "Central and peripheral routes to persuasion: application to advertising," in Advertising and Consumer Psychology, L. P. A. Woodside, Ed., pp. 3-23, Lexington Books, Lexington, Mass, USA, 1983.

[16] M. J. Dutta-Bergman, "Theory and practice in health communication campaigns: a critical interrogation," Health Communication, vol. 18, no. 2, pp. 103-122, 2005.

[17] M. D. Slater, "Specification and misspecification of theoretical foundations and logic models for health communication campaigns," Health Communication, vol. 20, no. 2, pp. 149-157, 2006.

[18] R. E. Petty and J. T. Cacioppo, "The elaboration likelihoodmodel of persuasion," Advances in Experimental Social Psychology, vol. 19, pp. 123-205, 1986.

[19] R. E. Petty, J. T. Cacioppo, A. J. Strathman, and J. R. Priester, “To think or not to think: exploring two routes to persuasion," in $\mathrm{Pu}$ rsuasion: Psychological Insights and Perspectives, S. Shavitt and T. C. Brock, Eds., pp. 113-147, Allyn and Bacon, Boston, Mass, USA, 1994. 
[20] B. Curbow, L. A. Fogarty, K. A. McDonnell, J. Chill, and L. B. Scott, "The role of physician characteristics in clinical trial acceptance: testing pathways of influence," Journal of Health Communication, vol. 11, no. 2, pp. 199-218, 2006.

[21] R. G. Campbell and A. S. Babrow, "The role of empathy in responses to persuasive risk communication: overcoming resistance to HIV prevention messages," Health Communication, vol. 16, no. 2, pp. 159-182, 2004.

[22] J. J. Igartua, L. Cheng, and O. Lopes, "To think or not to think: two pathways towards persuasion by short films on aids prevention," Journal of Health Communication, vol. 8, no. 6, pp. 513528, 2003.

[23] E. Rogers and J. Storey, "Communication campaigns," in Handbook of Communication Science, C. R. Berger and M. E. Chafee, Eds., pp. 419-445, Sage, Newbury Park, Calif, USA, 1987.

[24] H. N. Young and R. J. W. Cline, "Textual cues in direct-to-consumer prescription drug advertising: motivators to communicate with physicians," Journal of Applied Communication Research, vol. 33, no. 4, pp. 348-369, 2005.

[25] K. Witte and M. Allen, "When do scare tactics work? A metaanalysis of fear appeals," Health Education \& Behavior, vol. 27, pp. 608-632, 2000.

[26] M. T. Stephenson and K. Witte, "Generating fear in a risky world: creating effective risk messages," in Public Communication Campaigns, R. Rice and C. K. Atkin, Eds., pp. 88-102, Sage, Thousand Oaks, Calif, USA, 2001.

[27] K. Witte, "Putting the fear back into fear appeals: the extended parallel process model (EPPM)," Communication Monographs, vol. 61, pp. 113-134, 1994.

[28] D. J. OKeefe, "Elaboration likelihood model," in Persuasion: Theory \& Research, pp. 137-167, Sage, Thousand Oaks, Calif, USA, 2002.

[29] J. W. Carey, M. Morgan, and M. J. Oxtoby, "Intercoder agreement in analysis of responses to open-ended interview questions: examples from tuberculosis research," Cultural Anthropology Methods, vol. 8, no. 3, pp. 1-5, 1996.

[30] P. S. Sullivan, A. D. McNaghten, E. Begley, A. Hutchinson, and V. A. Cargill, "Enrollment of racial/ethnic minorities and women with HIV in clinical research studies of HIV medicines," Journal of the National Medical Association, vol. 99, no. 3, pp. 242-250, 2007.

[31] J. Gillman, J. Davila, and S. Sansgiry, "The effect of conspiracy beliefs and trust on HIV diagnosis, linkage, and retention in young MSM with HIV,' Journal of Health Care For the Poor and Underserved, vol. 24, no. 1, pp. 36-45, 2013.

[32] G. Corbie-Smith, S. B. Thomas, and D. M. M. St. George, "Distrust, race, and research," Archives of Internal Medicine, vol. 162, no. 21, pp. 2458-2463, 2002.

[33] C. Brach and I. Fraserirector, "Can cultural competency reduce racial and ethnic health disparities? A review and conceptual model," Medical Care Research and Review, vol. 57, supplement 1, pp. 181-217, 2000.

[34] J. I. McNeil, "A model for cultural competency in the HIV management of African American patients," Journal of the National Medical Association, vol. 95, 2, no. 2, pp. 3S-7S, 2003.

[35] K. K. Heavner, B. Shaw, H. Battles et al., HIV prevention fatigue: men who have sex with men (MSM) are tuning out HIV prevention messages, American Public Health Association, Washington, DC, USA, 2007.

[36] A. B. Bakker, "Persuasive communication about AIDS prevention: need for cognition determines the impact of message format," AIDS Education and Prevention, vol. 11, no. 2, pp. 150 $162,1999$.

[37] A. M. Oster, D. Pieniazek, X. Zhang et al., "Demographic but not geographic insularity in HIV transmission among young black MSM," AIDS, vol. 25, no. 17, pp. 2157-2165, 2011.

[38] C. Beyrer, P. Sullivan, J. Sanchez et al., “The global HIV epidemics in men who have sex with men (MSM): time to act," AIDS. In press.

[39] C. F. Kelley, E. S. Rosenberg, B. M. O’Hara et al., "Measuring population transmission risk for HIV: an alternative metric of exposure risk in men who have sex with men (MSM) in the US," PLoS One, vol. 7, no. 12, Article ID e53284, 2012.

[40] L. Bowleg, G. J. Burkholder, J. S. Massie et al., "Racial discrimination, social support, and sexual HIV risk among black heterosexual men," AIDS and Behavior, vol. 17, no. 1, pp. 407-418, 2013.

[41] M. Teti, A. E. Martin, R. Ranade et al., "'I'm a keep rising. I'm a keep going forward, regardless': exploring black men's resilience amid sociostructural challenges and stressors," Qualitative Health Research, vol. 22, no. 4, pp. 524-533, 2012.

[42] P. S. Sullivan, C. M. Khosropour, N. Luisi et al., "Bias in Online recruitment and retention of racial and ethnic minority men who have sex with men," Journal of Medical Internet Research, vol. 13, no. 2, article 38, 2011.

[43] S. N. Du Bois, S. E. Johnson, and B. Mustanski, "Examining racial and ethnic minority differences among YMSM during recruitment for an online HIV Prevention Intervention Study, AIDS and Behavior, vol. 16, no. 6, pp. 1430-1435, 2012.

[44] S. S. Bull, L. Lloyd, C. Rietmeijer, and M. McFarlane, "Recruitment and retention of an online sample for an HIV prevention intervention targeting men who have sex with men: the Smart Sex Quest Project," AIDS Care, vol. 16, no. 8, pp. 931-943, 2004.

[45] B. A. Koblin, K. H. Mayer, S. H. Eshleman et al., "Correlates of HIV aAcquisition in a cohort of black men who have sex with men in the United States: HIV prevention trials network (HPTN) 061," PLOS One, vol. 8, no. 7, Article ID e70413, 2013.

[46] R. W. Durant, R. B. Davis, D. M. M. St. George, I. C. Williams, C. Blumenthal, and G. M. Corbie-Smith, "Participation in research studies: factors associated with failing to meet minority recruitment goals," Annals of Epidemiology, vol. 17, no. 8, pp. 634-642, 2007.

[47] G. Corbie-Smith, W. C. Miller, and D. F. Ransohoff, "Interpretations of 'appropriate' minority inclusion in clinical research," American Journal of Medicine, vol. 116, no. 4, pp. 249-252, 2004.

[48] G. Corbie-Smith, S. Moody-Ayers, and A. D. Thrasher, "Closing the circle between minority inclusion in research and health disparities," Archives of Internal Medicine, vol. 164, no. 13, pp. 1362-1364, 2004. 

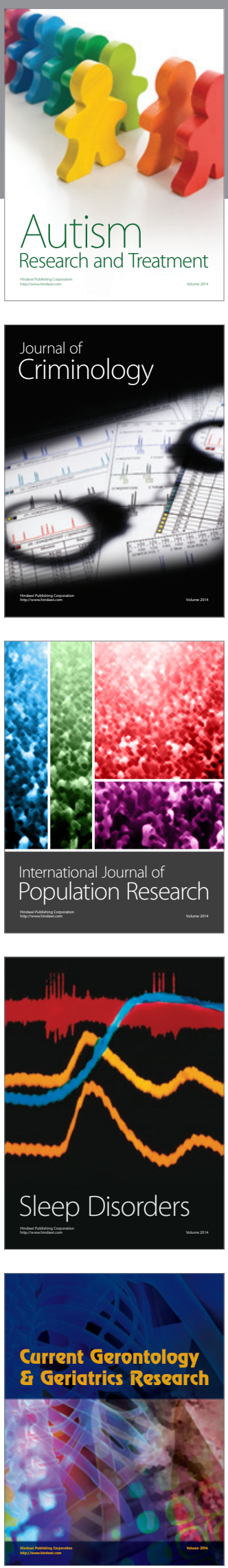
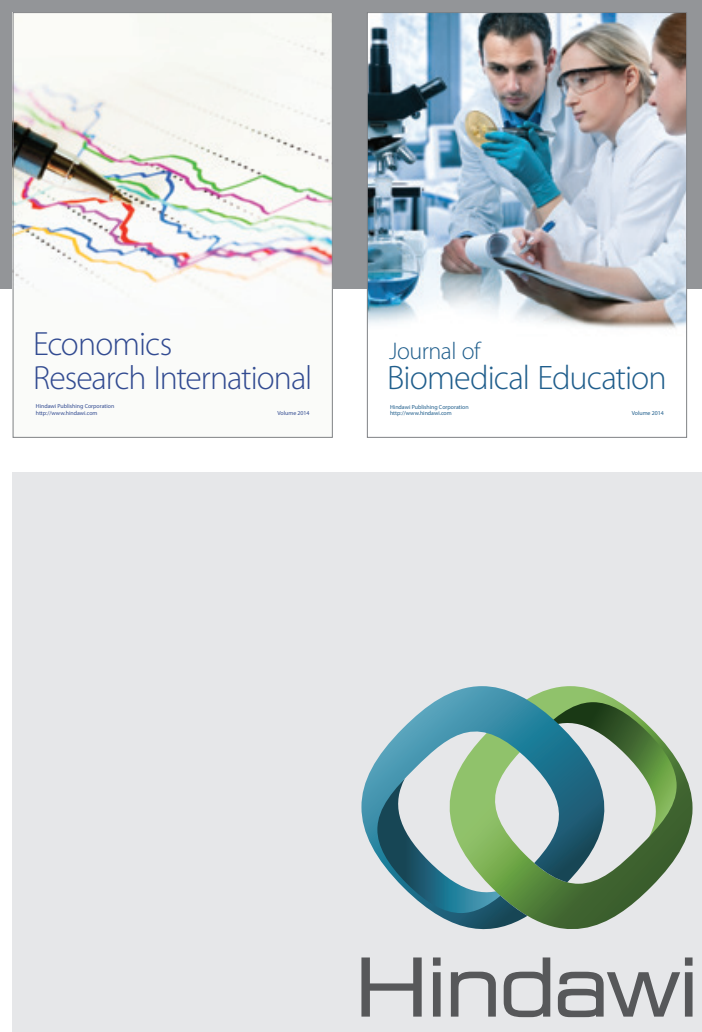

Submit your manuscripts at

http://www.hindawi.com
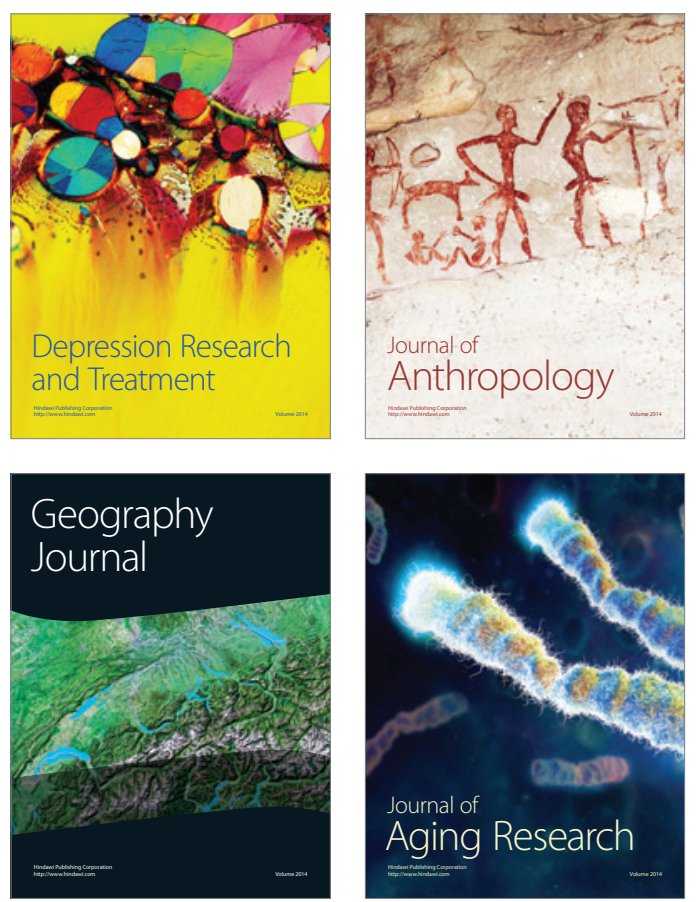
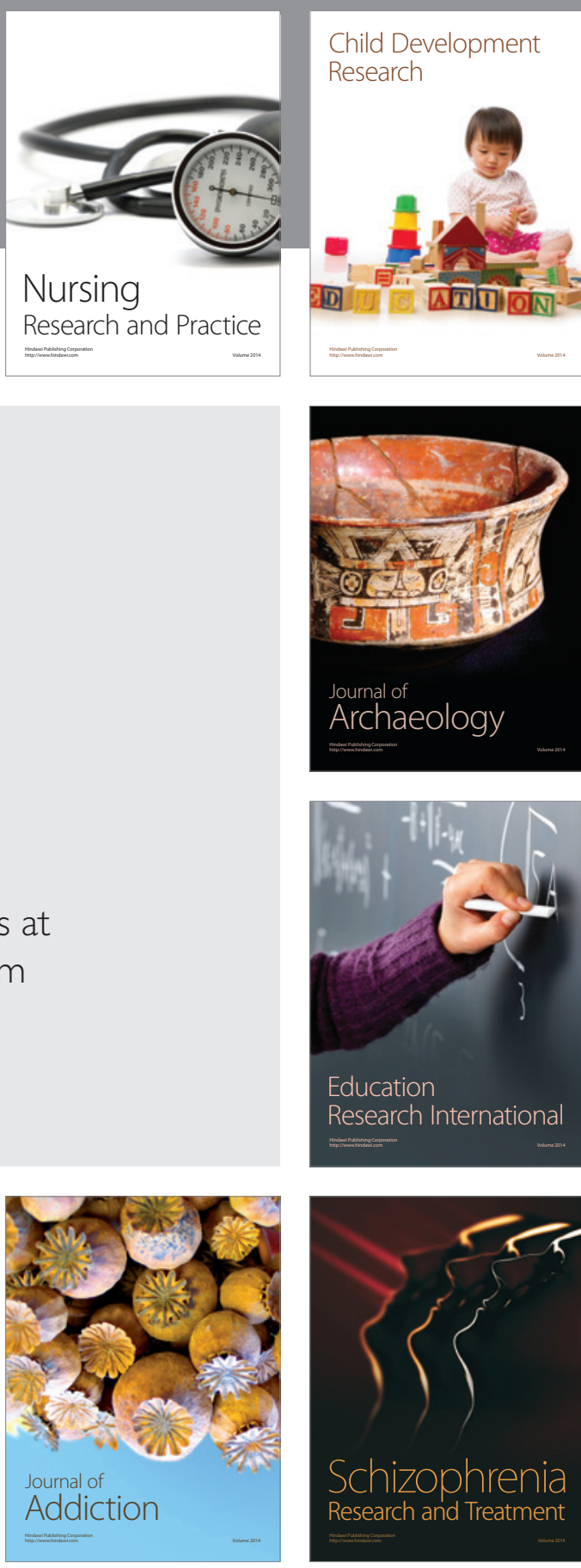

(D)
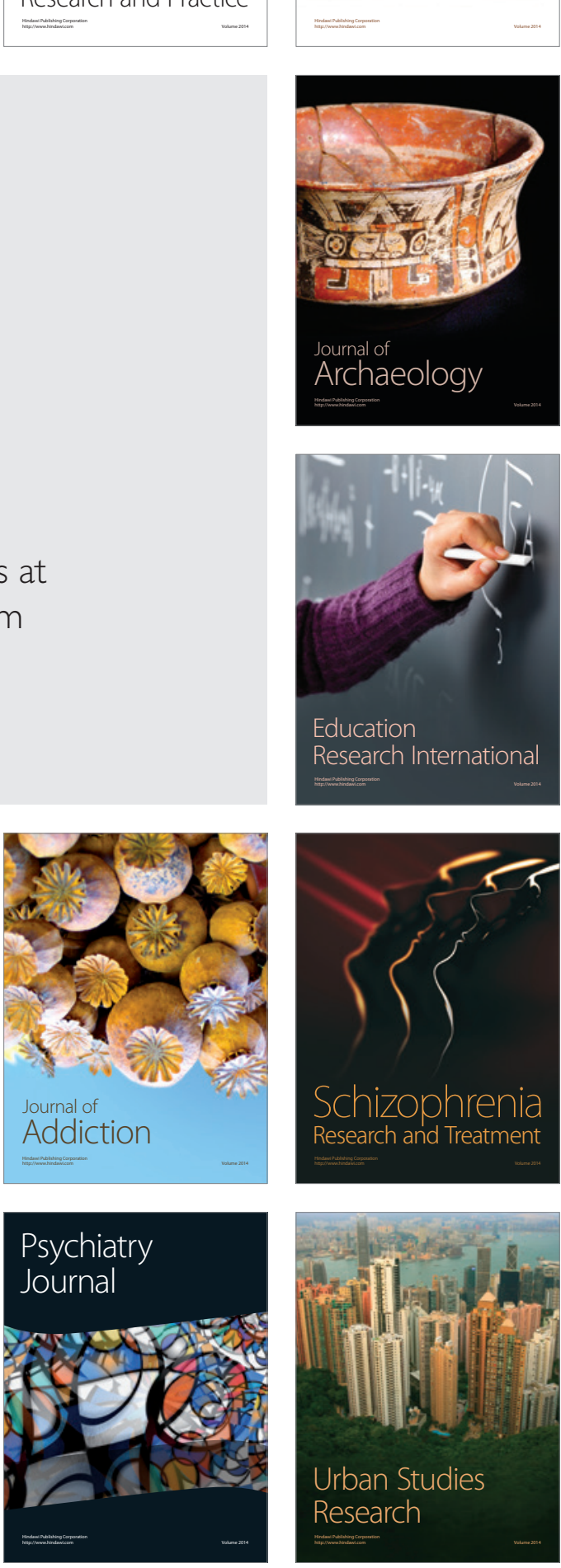